\title{
A novel discussion on centripetal force
}

\author{
Pramode Ranjan Bhattacharjee*†10 \\ ${ }^{1}$ Kabi Nazrul Mahavidyalaya, Sonamura, Tripura, India
}

Received on May 06, 2018; Revised on June 06, 2018; Accepted on June 12, 2018

\begin{abstract}
In order to enrich the field of Newtonian mechanics, some novel approaches for the derivation of the expression for the centripetal force have been offered in this paper. The paper starts with a lucid and easily readable discussion of how the centripetal force arises when a particle undergoes uniform circular motion and it ends with a systematic presentation of four analytical novel schemes in regard to the derivation of the expression for the centripetal force. Keywords: Classical mechanics, Circular motion, Centripetal force, Vector algebra.
\end{abstract}

\section{Introduction}

The concept of centripetal force introduced by Sir Isaac Newton falls within the purview of classical mechanics. According to Newton's first law of motion, every body continues to be in the state of rest or of uniform motion in a straight line except in so far as it be compelled by an external impressed force to change that state. If now a body moving with uniform speed along a circle is considered, then in order that this body should, as supposed, move in the same circular path, it must be acted upon by a constant force directed towards the centre of the circle. Such a force towards the centre, or central force, is called the centripetal force. Centripetal force may be regarded as the cause of circular motion. If the centripetal force ceases to exist, then the body instead of going round the curved path would go along the tangential path i.e. straight path with the speed available at that instant. Finding the source of centripetal force is an interesting problem. Centripetal force in case of planets going around the sun is due to the gravitational attraction between the earth and the sun. In case of an atomic electron moving round the nucleus in circular orbit, the centripetal force is due to the electrostatic force of attraction between the electron and the positively charged nucleus. For a charged particle moving round in a magnetic field, the Lorentz magnetic force plays the necessary role. When a vehicle moves round a curved path, the frictional force between the tyre and the ground provides the necessary centripetal force. When a stone tied to a thread is rotated in a circular path, the tension in the string would be the centripetal force.

A lot of discussions regarding centripetal force exist in traditional literature [1-14]. Most of them are concerned with qualitative as well as quantitative physics regarding the centripetal force.

\footnotetext{
*Endereço de correspondência: drpramode@rediffmail.com ${ }^{\dagger}$ Retired Principal
}

From the view point of academic interest as well as to enrich the traditional literature, this paper presents a novel discussion regarding the development of centripetal force acting on a particle moving round a circular path with uniform speed. That has been subsequently followed by offering four different novel techniques for the derivation of the expression for the centripetal force.

\section{Centripetal force at a glance}

Let us consider a particle moving in a circular track with uniform linear speed $v$. To describe once round the circular track, the particle will take a finite time. Now the entire motion during one time period may be thought of to take place by steps of infinitesimal displacements (Figure 1) starting from the point A (Initial position of the particle) up to the point $\mathrm{B}$ (say at the instant $\delta t$, where $\delta$ tis small). Then clearly the arc AB will be a small portion of the circular track and may be treated to be a straight line so that we can apply Newton's second law of motion for this elementary displacement of the particle. Now, at $A$, the linear momentum of the particle is $\mathbf{p}_{1}=m \mathbf{v}_{1}$ and at $B$ it is $\mathbf{p}_{2}=m \mathbf{v}_{2}$. Thus the change of momentum of the particle during this elementary displacement $=\mathbf{p}_{2}-\mathbf{p}_{1}=\mathbf{p}$ and it is along $\mathbf{C D}$. Let $\mathrm{Q}$ be the middle point of $\mathrm{AB}$.

Now, based on the limit process, the following approximations can be made.

$$
\lim _{\delta t \rightarrow 0} \operatorname{Angle}(B O Q)=\lim _{Q \rightarrow B} \operatorname{Angle}(B O Q)=0^{0} .
$$

As a result

$$
\lim _{\delta t \rightarrow 0} \text { Angle }(O Q B)=\lim _{\delta t \rightarrow 0} \text { Angle }(O B Q)=90^{\circ} .
$$

Again

$$
\lim _{\delta t \rightarrow 0} \text { Angle }(C B D)=\lim _{A \rightarrow B} \text { Angle }(C B D)=0^{0} .
$$




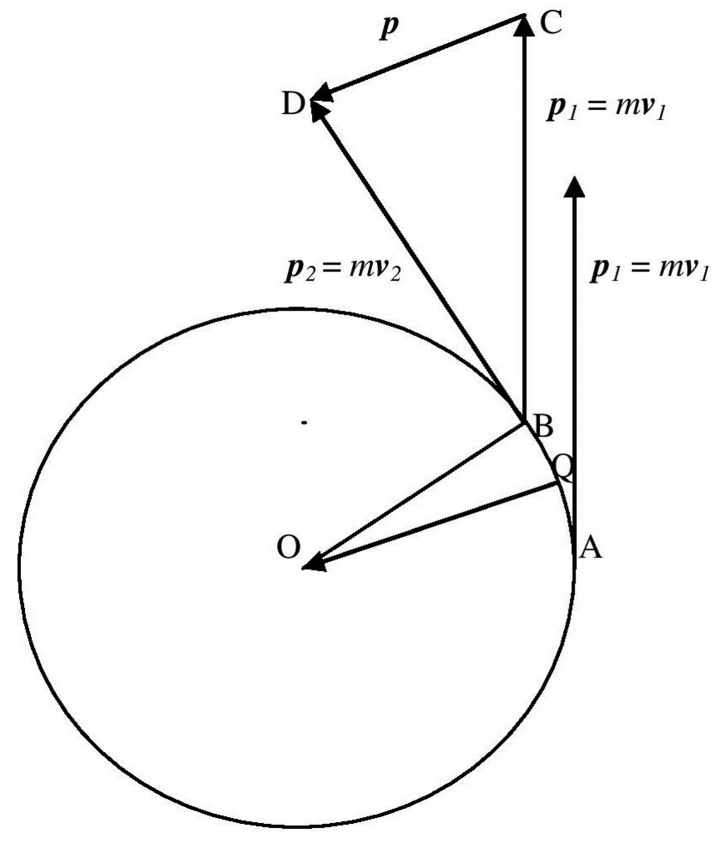

Figure 1: Diagram for explaining the concept of centripetal force.

Furthermore,

$\lim _{\delta t \rightarrow 0} Q C=\lim _{Q \rightarrow B} Q C=B C=\left|\mathbf{p}_{1}\right|=\left|m \mathbf{v}_{1}\right|=m v_{1}=m v$,

and

$$
\begin{aligned}
\lim _{\delta t \rightarrow 0} Q D & =\lim _{Q \rightarrow B} Q D=B D=\left|\mathbf{p}_{2}\right| \\
& =\left|m \mathbf{v}_{2}\right|=m v_{2}=m v .
\end{aligned}
$$

It thus follows from above that $Q C=Q D$. Hence Angle $(C D B)=\operatorname{Angle}(D C B)=90^{\circ}$. Further, here Angle $(O Q B)=90^{\circ}$. Hencee we have, Angle $(O Q B)=$ Angle $(C D B)$. But these are alternate angles since $A$ is very close to $B$. Hence $\mathbf{C D}$ is parallel to $\mathbf{Q O}$.

Thus the rate of change of momentum takes place along CD, i.e. along QO. By Newton's second law of motion the force acts on the particle along $\mathbf{Q O}$ since arc $\mathrm{AB}$ is of infinitesimal length, hence $\mathbf{Q O}, \mathbf{B O}$ and $\mathbf{A O}$ are all along the same direction.

It thus follows that during its elementary displacement from $\mathrm{A}$ to $\mathrm{B}$, the particle will be acted upon by a force directed towards the centre of the circular track. Since the entire circumference is the sum total of circular arcs of such elementary lengths it can be said that, at any instant of motion along the curved circular path, the particle is acted upon by a force directed towards the centre of the circle. This force is called centripetal force.

\section{Novel methods for the derivation of the expression for centripetal force}

In this section, several novel analytical methods in relation to the derivation of the expression for the centripetal force have been considered. It is felt that such methods will be interesting from the point of view of academic interest and they will enrich the relevant branch of study as well. From the view point of clarity and readability of presentation, it is worth mentioning here that in the following discussion concerning each of the four methods, a bold letter/alphabet/character has been used to represent a vector. On the other hand, a letter/alphabet/character which is not bold has been used to represent a scalar.

First method: Let us consider Figure 2 for deriving the expression for the centripetal force $\boldsymbol{F}$ acting on the particle revolving round the circular track of radius " $r$ " with uniform angular speed " $\omega$ ", the corresponding uniform linear speed being " $v$ ". Let initially (i.e. at $t=0$ ) the particle be at the point $\mathrm{A}$, the initial momentum of the particle being $\boldsymbol{p}_{0}=\mathrm{m} v_{1} \boldsymbol{n}_{1}$ and is clearly a constant vector. Let the momentum of the particle at any subsequent instant " $t$ ", when it reaches the position B of the circular track be $\boldsymbol{p}=m v_{2} \boldsymbol{n}_{2}$. It then clearly follows that $\boldsymbol{n}_{2}$ and hence $\boldsymbol{p}$ will be time dependent vectors.

The force $(\boldsymbol{F})$ acting on the particle at the instant " $t$ " is then given by,

$$
\boldsymbol{F}=\frac{d \boldsymbol{p}}{d t}=m \frac{d}{d t}\left(\boldsymbol{v}_{2}\right)
$$

or,

$$
\boldsymbol{F}=m\left(\boldsymbol{\omega} \times \boldsymbol{v}_{2}\right)
$$

since in this case, $\boldsymbol{v}_{2}=\boldsymbol{\omega} \times \boldsymbol{r}_{2}$, and the angular velocity $\boldsymbol{\omega}$ is a constant vector.

Equation (1) gives the vectorial expression for the force acting on the particle at the instant " $t$ " when the particle is at B. Clearly this force on the particle when it is at B is directed along BO. Thus the force acting on the particle, undergoing uniform circular motion, at any instant is

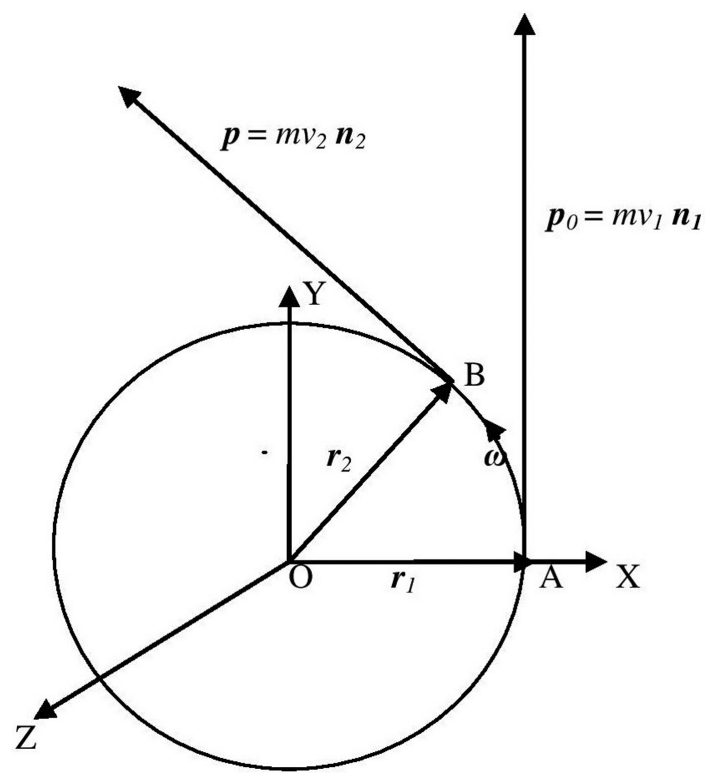

Figure 2: Diagram used for deriving the expression for the centripetal force by the first method. 
directed towards the centre of the circle. Such a force is called the centripetal force and its magnitude can be obtained from equation (1) as

$$
F=|\boldsymbol{F}|=\left|m\left(\boldsymbol{\omega} \times \boldsymbol{v}_{2}\right)\right|=m \omega v,
$$

where $v$ is the uniform linear speed of the particle. Or,

$$
F=\frac{m v^{2}}{r}
$$

since here, $\omega=\frac{v}{r}$.

The corresponding centripetal acceleration of the particle will then be equal to $\frac{v^{2}}{r}$.

Second method: As shown in Figure 3, let the particle moving with uniform speed go from A to B along the circular track of radius " $r$ " during a small period of time $\delta t$, so that the angle $\delta \theta$ subtended at the centre of the circular track by the arc AB is small.

Change of velocity of the particle during the period $\delta t$ is given by, $\boldsymbol{R}=\boldsymbol{v}_{2}-\boldsymbol{v}_{1}=v \boldsymbol{n}_{2}-v \boldsymbol{n}_{1}$.

Then we have,

$$
R^{2}=\left(v \boldsymbol{n}_{2}-v \boldsymbol{n}_{1}\right) \cdot\left(v \boldsymbol{n}_{2}-v \boldsymbol{n}_{1}\right) \approx 4 v^{2}\left(\frac{\delta \theta}{2}\right)^{2}
$$

using the approximation, $\sin \frac{\delta \theta}{2} \approx \frac{\delta \theta}{2}$, since $\delta \theta$ is small.

Hence, $R=v \delta \theta$ or, $\boldsymbol{R} \approx(v \delta \theta) \boldsymbol{s}$, where $\boldsymbol{s}$ a unit vector along the direction of $\boldsymbol{R}$.

The acceleration $(\boldsymbol{f})$ of the particle will be given by,

$$
\boldsymbol{f}=\frac{\boldsymbol{R}}{\delta t}=\frac{[(v \delta \theta) \boldsymbol{s}]}{\delta t}=v \omega \boldsymbol{s}
$$

where $\omega$ is the uniform angular speed of the particle.

Again considering Figure 3, it can be readily seen that, $\phi=90^{\circ}-\frac{\delta \theta}{2} \approx 90^{\circ}$, since $\delta \theta$ is small.

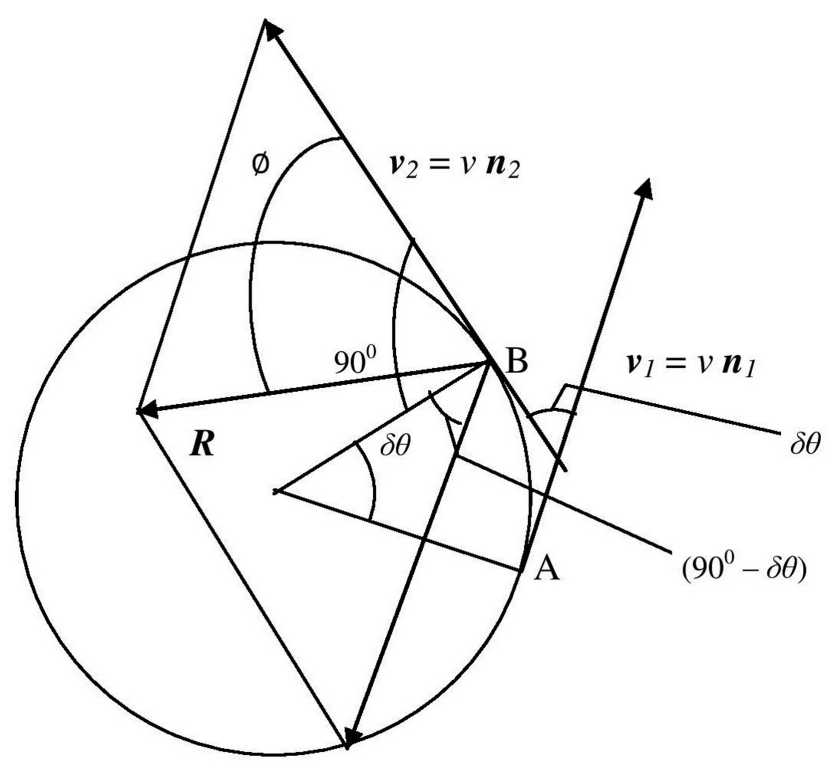

Figure 3: Diagram used for the derivation of the expression for the centripetal force in the second and third methods.
This implies that $\boldsymbol{s}$, and hence $\boldsymbol{f}$ must be directed towards the centre of the circular track. The force acting on the particle is then given by, $\boldsymbol{F}=m v \omega s$, and this force is directed towards the centre of the circular track. Such a force is well-known as the centripetal force.

The magnitude of the centripetal force $(\boldsymbol{F})$ acting on the particle will then be given by,

$$
F=m v \omega=\frac{m v^{2}}{r}
$$

since $\omega=\frac{v}{r}$.

Third method: Let us consider the uniform circular motion of a particle described by Figure 3. The acceleration $(\boldsymbol{f})$ of the particle moving round the circular track with uniform linear speed $v$ at any instant is given by

$$
\boldsymbol{f}=\frac{d \boldsymbol{v}}{d t}=\lim _{\delta t \rightarrow 0}\left[\frac{\left\{v \boldsymbol{n}_{2}-v \boldsymbol{n}_{1}\right\}}{\delta t}\right],
$$

where $\boldsymbol{n}_{2}$ and $\boldsymbol{n}_{1}$ are respectively unit vectors along the directions of the linear velocities of the particle at the instants " $t+\delta t$ " and " $t$ ".

Also,

$$
\boldsymbol{f}=v \boldsymbol{s} \lim _{\delta t \rightarrow 0}\left(\frac{\delta \theta}{\delta t}\right)=v \boldsymbol{s} \omega=\left(\frac{v^{2}}{r}\right) \boldsymbol{s},
$$

where $\boldsymbol{s}$ is a unit vector along the direction of $\left(\boldsymbol{n}_{2}-\boldsymbol{n}_{1}\right)$, and $\omega$ is the uniform angular speed of the particle.

Again considering Figure 3, it follows that, $\phi=90^{\circ}-\frac{\delta \theta}{2} \approx 90^{\circ}$, since $\delta \theta$ is small.

This implies that the direction of $\boldsymbol{s}$, and hence $\boldsymbol{f}$ must pass through the centre of the circular track. The force acting on the particle is then given by, $\boldsymbol{F}=m\left(\frac{v^{2}}{r}\right) \mathbf{s}$. This force acting towards the centre of the circular track is the well-known centripetal force.

The magnitude of the centripetal force $(\boldsymbol{F})$ acting on the particle will then be given by, $F=\frac{m v^{2}}{r}$.

Fourth method: With reference to Figure 4, the position vector of the particle when it is at $\mathrm{P}$ at the instant $t$ is given by $\boldsymbol{r}=r \cos \theta \boldsymbol{i}+r \sin \theta \boldsymbol{j}$.

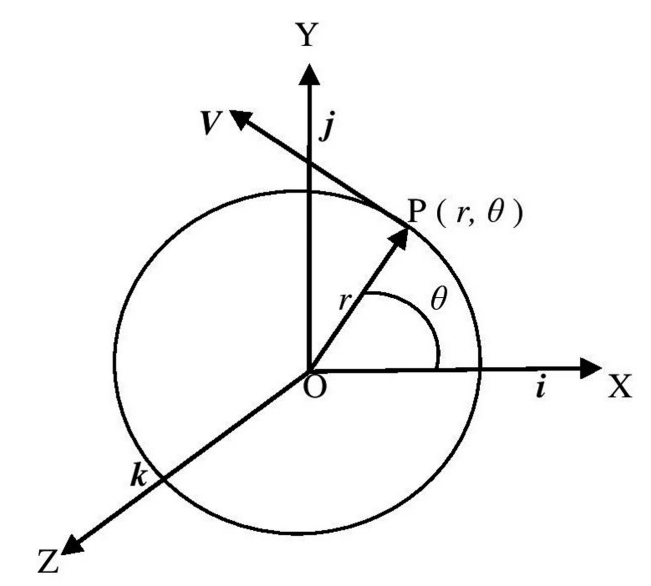

Figure 4: Diagram for the derivation of the expression for the centripetal force by fourth method. 
Velocity of the particle at the instant $t$ is then

$$
\boldsymbol{v}=\frac{d \boldsymbol{r}}{d t}=-r \sin \theta \frac{d \theta}{d t} \boldsymbol{i}+r \cos \theta \frac{d \theta}{d t} \boldsymbol{j}
$$

since $\boldsymbol{r}, \boldsymbol{i}$, and $\boldsymbol{j}$ are constants being independent of $t$.

The acceleration $(\boldsymbol{f})$ of the particle at the instant $t$ when it is at the point $\mathrm{P}$ is then

$$
\boldsymbol{f}=\frac{d \boldsymbol{v}}{d t}=-r \cos \theta\left(\frac{d \theta}{d t}\right)^{2} \boldsymbol{i}-r \sin \theta\left(\frac{d \theta}{d t}\right)^{2} \boldsymbol{j}
$$

since $r$ and $\frac{d \theta}{d t}$ are constants, both being independent of $t$. Then $\boldsymbol{f}=-\left(\frac{d \theta}{d t}\right)^{2} r \boldsymbol{r}_{0}$, where $\boldsymbol{r}_{0}$ is a unit vector along the direction of $\boldsymbol{r}$.

The magnitude of the acceleration of the particle at the instant $t$ when it is at $\mathrm{P}$ is then $f=r\left(\frac{d \theta}{d t}\right)^{2}=r \omega^{2}=\frac{v^{2}}{r}$, since the angular speed is $\omega=\frac{v}{r}$.

The said acceleration is directed along $-\boldsymbol{r}_{0}$, i.e. towards the centre of the circle. This acceleration is called the centripetal acceleration.

The magnitude of the corresponding centripetal force $(\boldsymbol{F})$ acting on the particle at the instant $t$ is then given by $F=\frac{m v^{2}}{r}$.

\section{Conclusion}

This paper deals with a novel discussion on the development of the centripetal force acting on a body moving round a circular track with uniform speed. It also offers four novel procedures for the derivation of the expression for the centripetal force that make use of different mathematical tools. Because of its novelty in respect of the qualitative and quantitative physics incorporated, the present work will enrich the relevant field of study. At the same time, it will also be interesting from the view point of academic interest. The author feels that this paper will be interesting to the engineering as well as the science students at undergraduate level in respect of getting them refreshed about the relevant topic.

\section{References}

[1] D. Halliday, R. Resnick and J. Walker, Principles of Physics (Wiley India, New Delhi, 2013), $9^{\text {th }}$ ed.

[2] R.G. Takwale and P.S. Puranik, Introduction to Classical Mechanics (Tata McGraw-Hill Publishing, New Delhi, 2009).

[3] H. Crew, General Physics, an elementary textbook for colleges (The Macmillan Company, New York, 1908).

[4] D.S. Mathur, Elements of general properties of matter (Reprint, S. Chand \& Co., New Delhi, 2010).

[5] E. Edser, General Physics for students (Macmillan and Co., London, 1911).

[6] A.P. French, Newtonian Mechanics (Thomas Nelson \& Sons Ltd., London, 1971).

[7] D.B. Sinha and J.M. Das Sharma, College Physics, Vol. I (Modern Book Agency Private limited, Calcutta, 1976).
[8] A.R. Basu, A text book of Engineering Mechanics (Dhanpat Rai \& Co., Delhi, 2002).

[9] R.K. Gaur and S.L. Gupta, Engineering Physics (Dhanpat Rai Publications, New Delhi, 2011).

[10] R.C. Brown, Mechanics and Properties of matter (Longmans Green and Company, London, 1959).

[11] H.C. Verma, Concepts of Physics, Vol. 1 (Print, Bharati Bhawan, Patna, 2013).

[12] R.S. Khurmi, A textbook of Engineering Mechanics (S. Chand \& Co., New Delhi, 2013), $20^{\text {th }}$ ed.

[13] I. Newton and R.C. Henry, American Journal of Physics 68, 637 (2000).

[14] I.M. Freeman, American Journal of Physics 30, 421 (1962). 